\title{
More Properties of Semi-Linear Uniform Spaces
}

\author{
S. A. Alhihi \\ Department of Mathematics, Al-Balqa' Applied University, Alsalt, Jordan \\ Email: suad.hihi@bau.edu.jo, suadalhihi@yahoo.com
}

Received 22 April 2015; accepted 7 June 2015; published 10 June 2015

Copyright (C 2015 by author and Scientific Research Publishing Inc.

This work is licensed under the Creative Commons Attribution International License (CC BY). http://creativecommons.org/licenses/by/4.0/

c) (i) Open Access

\begin{abstract}
In this paper we shall generalize the definition given in [1] for Lipschitz condition and contractions for functions on a non-metrizable space, besides we shall give more properties of semi-linear uniform spaces.
\end{abstract}

\section{Keywords}

Lipschitz Condition, Contractions, Not Metrizable Spaces, Semi-Linear Spaces, Uniform Spaces, Fixed Point

\section{Introduction}

The notion of uniformity has been investigated by several mathematician as Weil [2]-[4], Cohen [5] [6], and Graves [7].

The theory of uniform spaces was given by Burbaki in [8]. Also Wiels in his booklet [4] defined uniformly continuous mapping. For more information about Uniform spaces one my refer to [9].

In 2009, Tallafha, A. and Khalil, R. [10], defined a new type of uniform spaces, namely semi-linear uniform spaces and they gave example of semi-linear space which was not metrizable. Also they defined a set valued map $\rho$ on $X \times X$, by which they studied some cases of best approximation in such spaces. More precisely, they gave the following.

Let $(X, \Gamma)$ be a semi-linear uniform space; $E \subseteq X$ is proximinal if for any $x \in X$, there exists $e \in E$ such that $\rho(x, E)=\rho(x, e)$. They asked that "must every compact is proximinal", they gave the answer for the cases-i) $E$ is finiate; ii) If $x_{n}$ converges to $x$, then $\left\{x, x_{1}, x_{2}, \cdots\right\}$ is proximinal.

In [11], Tallafha, A. defined another set valued map $\delta$ on $X \times X$, and gave some properties of semi-linear uniform spaces using the maps $\rho$ and $\delta$. Also in [1] [12], Tallafha defined Lipschitz condition and contractions for functions on semi-linear uniform spaces, which enabled us to study fixed point for such functions. 
Lipschitz condition, and contractions are usually discussed in metric and normed spaces and never been studied in other weaker spaces. We believe that the structure of semi-linear uniform spaces is very rich, and all the known results on fixed point theory can be generalized.

The object of this paper is to generalize the definition of Lipschitz condition, and contraction mapping on semi-linear uniform spaces given by Tallafha [12]. Also we shall give a new topopological properties and more properties of semi-linear uniform spaces.

\section{Semi-Linear Uniform Space}

Let $X$ be a set and $D_{X}$ be a collection of subsets of $X \times X$, such that each element $V$ of $D_{X}$ contains the diagonal $\Delta=\{(x, x): x \in X\}$, and $V=V^{-1}=\{(y, x):(x, y) \in V\}$ for all $V \in D_{X}$ (symmetric). $D_{X}$ is called the family of all entourages of the diagonal. if,

Definition 1 [10]. Let $\Gamma$ be a sub collection of $D_{X}$, the pair $(X, \Gamma)$ is called a semi-linear uniform space

i) $\Gamma$ is a chain.

ii) For every $V \in \Gamma$, there exists $U \in \Gamma$ such that $U \circ U \subseteq V$.

iii) $\bigcap_{V \in \Gamma} V=\Delta$.

iv) $\bigcup_{V \in \Gamma} V=X \times X$

Definition 2 [10]. Let $(X, \Gamma)$ be a semi-linear uniform space, for $(x, y) \in X \times X$, let $\Gamma_{(x, y)}=\{V \in \Gamma:(x, y) \in V\}$. Then, the set valued map $\rho$ on $X \times X$ is defined by $\rho(x, y)=\bigcap_{V \in \Gamma_{(x, y)}} V$.

Clearly for all $(x, y) \in X \times X$, we have $\rho(x, y)=\rho(y, x)$ and $\Delta \subseteq \rho(x, y)$. Let

$\Gamma \backslash \Gamma_{(x, y)}=\left(\Gamma_{(x, y)}\right)^{c}=\{V \in \Gamma:(x, y) \notin V\}$, from now on, we shall denote $\Gamma \backslash \Gamma_{(x, y)}$ by $\Gamma_{(x, y)}^{c}$.

Definition 3 [11]. Let $(X, \Gamma)$ be a semi-linear uniform space. Then, the set valued map $\delta$ on $X \times X$ is defined by, $\delta(x, y)= \begin{cases}\bigcup_{V \in \Gamma_{(x, y)}^{c}} V & \text { if } x \neq y, \\ \phi & \text { if } x=y .\end{cases}$

The following results are given in [12].

Proposition 1. Let $(X, \Gamma)$ be a semi-linear uniform space, and $\Lambda$ is a sub collection of $\Gamma$, then $\bigcap_{V \in \Lambda} V \neq \Delta$, if an only if there exist $U \in \Gamma$ such that $U \varsubsetneqq \bigcap_{V \in \Lambda} V$.

Corollary 1. Let $(X, \Gamma)$ be a semi-linear uniform space. If $\rho(x, y) \neq \Delta$, then,

1) There exist $U \in \Gamma$ such that $U \varsubsetneqq \rho(x, y)$.

2) $U \subseteq \delta(x, y)$.

Let $V \in D_{X}$ the family of all entourages of the diagonal, then for all $n \in \mathbb{N}$, by $n V$, we mean $V \circ V \circ \cdots \circ V$ $n$-times and $0 V=\Delta$, so for all $V \in D_{X}, V \subseteq n V$ and $n V \in D_{X}$ for all $n \in \mathbb{N}$.

Proposition 2. Let $(X, \Gamma)$ be a semi-linear uniform space. If $x \neq y$, then $n \delta(x, y)=\bigcup_{V \in \Gamma_{(x, y)}^{c}} n V$.

Proposition 3. Let $(X, \Gamma)$ be a semi-linear uniform space. If $\Lambda$ is a sub collection of $\Gamma$, then $\bigcup_{V \in \Lambda} n V=n \bigcup_{V \in \Lambda} V$.

Question. Does $\bigcap_{V \in \Gamma(x, y)} n V \subseteq n \rho(x, y)$ ?

\section{Topological Properties of Semi-Linear Uniform Spaces}

Definition 4 [13]. For $x \in X$ and $V \in \Gamma$. The open ball of center $x$ and radius $V$ is defined by $B(x, V)=\{y:(x, y) \in V\}$, equivalently $B(x, V)=\{y: \rho(x, y) \subseteq V\}$. 
Clearly if $y \in B(x, V)$, then there is a $W \in \Gamma$ such that $B(y, W) \subseteq B(x, V)$. So $\{B(x, V), x \in X, V \in \Gamma\}$ is a base for some topology on $X$. This topology is denoted by $\tau_{\Gamma}$.

More presicly $\tau_{\Gamma}=\{G \subseteq X$ : for all $x \in G$, there exist $V \in \Gamma$, such that $B(x, V) \subseteq G\}$.

In [10] it is shown that $\tau_{\Gamma}$ is Hausdorf, so if $X$ is finite then we have the discreet topology, therefore interesting examples are when $X$ is infinite. Also, if $X$ is infinite then $\Gamma$ should be infinite, other wise $\Delta \in \Gamma$, which implies also that the topology is the discrete topology.

Proposition 4. Let $(X, \Gamma)$ be a semi-linear uniform space and $\Gamma^{\prime}$ a subcollection of $\Gamma$ satisfies,

i) For every $U \in \Gamma$, there is a $V \in \Gamma^{\prime}$ such that $V \subseteq U$.

ii) $\bigcup_{V \in \Gamma^{\prime}} V=X \times X$.

iii) $\bigcap_{V \in \Gamma^{\prime}} V=\Delta$.

Then $\left(X, \Gamma^{\prime}\right)$ is a semi-linear uniform space and $\tau_{\Gamma}=\tau_{\Gamma^{\prime}}$.

Proof. Since $\Gamma^{\prime}$ is a subcollection of $\Gamma$, then $\Gamma^{\prime}$ is a subcollection of $D_{X}$ and i), iii), iv) in definition (1.1) are satisfied. Now for $U^{\prime}, V^{\prime} \in \Gamma^{\prime}$, ther exist $U \in \Gamma$ such that $U \subseteq\left(U^{\prime} \cap V^{\prime}\right)$, so there is a $W \in \Gamma^{\prime}$ such that $W \subseteq U \subseteq\left(U^{\prime} \cap V^{\prime}\right)$. So $\left(X, \Gamma^{\prime}\right)$ is a semi-linear uniform space. Now $\tau_{\Gamma^{\prime}} \subseteq \tau_{\Gamma}$ is clear. Let $O$ be a nonempty open set in $\tau_{\Gamma}$, then if $x \in O$ ther exist $U \in \Gamma$ such that $B(x, U) \subseteq O$. Let $V \in \Gamma^{\prime}$ such that $V \subseteq U$, so $B(x, V) \subseteq O$, hence $\tau_{\Gamma} \subseteq \tau_{\Gamma^{\prime}}$.

Theorem 1. Let $(X, \Gamma)$ be a semi-linear uniform space and $\tau_{\Gamma}$ the topology on $X$ indused by $\Gamma$, then.

i) $\Gamma$ can be consider as asubset of $\tau_{\Gamma} \times \tau_{\Gamma}$.

ii) Fore all $x, y \in X, \delta(x, y) \in \tau_{\Gamma} \times \tau_{\Gamma}$.

Proof. i). Let $\Gamma^{\prime}=\left\{U^{\circ}: U \in \Gamma\right\}$, where $U^{\circ}$ is the interior of $U$ with respect to the topology $\tau_{\Gamma} \times \tau_{\Gamma}$ on $X \times X$. By Proposition 2.2, $\left(X, \Gamma^{\prime}\right)$ is a semi-linear uniform space indusing the topology $\tau_{\Gamma}$ on $X \times X$. Sine we deal with the toplogy $\tau_{\Gamma}$, we may replace $\Gamma$ with $\Gamma^{\prime}$, so for all $V \in \Gamma^{\prime}, V=U^{\circ}$ for some $U \in \Gamma$, hence $V \in \tau_{\Gamma} \times \tau_{\Gamma}$.

ii) Is clear by definition of $\delta(x, y)$.

In [11], Tallafha gave some important properties of semi-linear uniform spaces, using the set valued map $\rho$ and $\delta$.

Now we shall give more properties of semi-linear uniform spaces.

\section{More Properties of Semi-Linear Uniform Spaces}

Let $(X, \Gamma)$ be a semi-linear uniform space, then $\Gamma$ is a chain so there exist a well order set $(\mathbf{R}, \preceq)$, such that $\Gamma=\left\{U_{r}, r \in \mathbf{R}\right\}$. For $x, y \in X, x \neq y$, then, ther exist $U \in \Gamma$, such that $(x, y) \in U$. That is, there exist $r_{0} \in \mathbf{R}$ such that $(x, y) \in U_{r_{0}}$. This implies, if $(x, y) \notin U_{r}$ then $U_{r} \subseteq U_{r_{0}}$. Hence $\mathbf{S}=\left\{r \in \mathbf{R}:(x, y) \notin U_{r}\right\}$ is bounded above by $r_{0}$. By Zorn's Lemma $\mathbf{S}$ has a maximal element $r_{(x, y)}$. So $\delta(x, y) \subseteq U_{r_{(x, y)}} \subseteq \rho(x, y)$. This copleet the proof of the following lemma.

Lemma 1. Let $(X, \Gamma)$ be a semi-linear uniform space, then $\Gamma=\left\{U_{r}, r \in \mathbf{R}\right\}$, where $\mathbf{R}$ is a well order set, then for $x, y \in X, \quad x \neq y$, ther exist $r_{(x, y)} \in \mathbf{R}$, such that $\delta(x, y) \subseteq U_{r_{(x, y)}} \subseteq \rho(x, y)$.

Remember that for all $V \in D_{X}$ the family of all entourages of the diagonal, $V$ satisfies the following nice properties, $V \subseteq n V$ and $n V \in D_{X}$ for all $n \in \mathbb{N}$. Let $\Lambda, \delta$ and $\rho$ be a subcollections of $D_{X}$, defined by $\delta=\{\delta(x, y): x, y \in X, x \neq y\}, \quad \rho=\{\rho(x, y): x, y \in X, x \neq y\}$ and $\Lambda=\delta \cup \rho \cup \Gamma$. For all $A \in \Lambda$, by Corollary 1.6, there exist $U \in \Gamma$ such that $U \subseteq A$. So we can define $\frac{1}{n} A$, for an element $A \in \Lambda$ by.

Definition 5. For $n \in \mathbb{N}$ and $A \in \Lambda$. Define $\frac{1}{n} A$ by,

$$
\frac{1}{n} A=\bigcup_{U \in \Gamma}\{U: n U \subseteq A\}
$$

Clearly $\frac{1}{n} \Delta=\Delta$ and $\frac{1}{n} A \in D_{X}$ for all $A \in \Lambda$. But $\frac{1}{n} A$ need not be an element in $\Gamma$, evin if $A \in \Gamma$. 
But we have.

Lemma 2. Let $(X, \Gamma)$ be a semi-linear uniform space, $\Gamma=\left\{U_{r}, r \in \mathbf{R}\right\}, \quad \mathbf{R}$ is a well order set, and $A \in \Lambda$.

Then there exist $r_{A} \in \mathbf{R}$, such that $\frac{1}{n} A \subseteq U_{r_{A}}$ and if $r \in \mathbf{R}$ satisfied $r_{A} \prec r$, then $n U_{r} \backslash A \neq \phi$.

Proof. For $A \in \Lambda$, there exist $r_{0} \in \mathbf{R}$ such that $A \subseteq U_{r_{0}}$, so for every $U \in \Gamma$ satisfies $n U \subseteq A$, we have $U \subseteq U_{r_{0}}$. Hence $T=\left\{r \in \mathbf{R}: n U_{r} \subseteq A\right\}$ is bounded above by $r_{0}$. Hence $r_{A}$ be the maximal element of $T$.

Theorem 2. Let $A \in \Lambda$, and $\sigma$ a subcolection of $\Lambda$. For $n \in \mathbb{N}$, we have

i) $n\left(\frac{1}{n} A\right) \subseteq A$.

ii) If $B \in \Gamma$ satisfies $n B \subseteq A$, then $B \subseteq \frac{1}{n} A$.

iii) $\frac{1}{n+1} A \subseteq \frac{1}{n} A$

iv) $\frac{1}{n} A \subseteq A$.

v) $\frac{1}{n} \bigcap_{A \in \sigma} A=\bigcap_{A \in \sigma} \frac{1}{n} A$.

vi) $\bigcup_{A \in \sigma} \frac{1}{n} A \subseteq \frac{1}{n} \bigcup_{A \in \sigma} A$.

Proof. i). Let $\left(s_{0}, s_{n}\right) \in n\left(\frac{1}{n} A\right)$, then there exist $s_{1}, \cdots, s_{n-1}$ such that $\left(s_{j}, s_{j+1}\right) \in\left(\frac{1}{n} A\right), j=0,1, \cdots, n-1$.

So there exist $U_{1}, U_{2}, \cdots, U_{n}$ in $\Gamma$ such that $\left(s_{j-1}, s_{j}\right) \in U_{j}$ and $n U_{j} \subseteq A, j=1, \cdots, n$. Since $\Gamma$ is a chain, there exist $j_{0} \in\{1, \cdots, n\}$ such that $U_{j} \subseteq U_{j_{0}}$ for all $j=1, \cdots, n$. So $\left(s_{0}, s_{n}\right) \in n U_{j_{0}} \subseteq A$.

ii) and iv), are clear by definition of $\frac{1}{n} A$.

iii) $\frac{1}{n+1} A=\bigcup_{U \in \Gamma}\{U:(n+1) U \subseteq A\} \subseteq \bigcup_{U \in \Gamma}\{U: n U \subseteq A\}=\frac{1}{n} V$. v) $\frac{1}{n} \bigcap_{A \in \sigma} A \subseteq \frac{1}{n} A$, for all $A \in \sigma$, so $\frac{1}{n} \bigcap_{A \in \sigma} A \subseteq \bigcap_{A \in \sigma} \frac{1}{n} A$. Conversly, Let $\left(s_{0}, s_{n}\right) \in \bigcap_{A \in \sigma} \frac{1}{n} A$, then, for all $A \in \sigma$, there exist $s_{1}, \cdots, s_{n-1}$ such that $\left(s_{j}, s_{j+1}\right) \in \frac{1}{n} A, j=0,1, \cdots, n-1$. So there exist $U_{1}, U_{2}, \cdots, U_{n}$ in $\Gamma$ such that $\left(s_{j-1}, s_{j}\right) \in U_{j}$ and $n U_{j} \subseteq A$, $j=1, \cdots, n$. Since $\Gamma$ is a chain, there exist $j_{0} \in\{1, \cdots, n\}$ such that $U_{j} \subseteq U_{j_{0}}$ for all $j=1, \cdots, n$ So $\left(s_{0}, s_{n}\right) \in n U_{j_{0}} \subseteq A$.

Let $n \in \mathbb{N}$ and $A_{\alpha} \in D_{X}, \quad \alpha \in \Lambda$, replacing $A$ by $\bigcup_{\alpha \in \Lambda} A_{\alpha}$, or by $\bigcap_{\alpha \in \Lambda} A_{\alpha}$, we have.

Corollary 2. For $x, y \in X$ where $(X, \Gamma)$ is a semi-linear uniform spaces, we have,

i) $\frac{1}{n} \delta(x, y)=\left\{\begin{array}{ll}\bigcap_{V \in \Gamma_{(x, y)}} \frac{1}{n} V & \text { if } x \neq y \\ \phi & \text { if } x=y\end{array}\right.$.

ii) $\frac{1}{n} \rho(x, y)=\frac{1}{n} \bigcap_{V \in \Gamma_{(x, y)}} V \subseteq \bigcap_{V \in \Gamma_{(x, y)}} \frac{1}{n} V$.

Corollary 3. Let $n \in \mathbb{N}$ and $A_{\alpha} \in D_{X}, \quad \alpha \in \Lambda$, then i) $n\left(\frac{1}{n} \bigcup_{\alpha \in \Lambda} A_{\alpha}\right) \subseteq \bigcup_{\alpha \in \Lambda} A_{\alpha}$. 
ii) If $B \in V$ satisfies $n B \subseteq\left(\bigcup\left\{A_{\alpha}: \alpha \in \Lambda\right\}\right)$, then $B \subseteq \frac{1}{n} \bigcup\left\{A_{\alpha}: \alpha \in \Lambda\right\}$.

iii) $\frac{1}{n+1} \bigcap_{\alpha \in \Lambda} A_{\alpha} \subseteq \frac{1}{n} \bigcap_{\alpha \in \Lambda} A_{\alpha}$.

iv) $n\left(\frac{1}{n} \bigcap_{\alpha \in \Lambda} A_{\alpha}\right) \subseteq \bigcap_{\alpha \in \Lambda} A_{\alpha}$.

v) If $V \in D_{X}$ satisfies $n B \subseteq \bigcup_{\alpha \in \Lambda} A_{\alpha}$, then $B \subseteq \frac{1}{n} \bigcup_{\alpha \in \Lambda} A_{\alpha}$.

vi) $\frac{1}{n+1}\left(\bigcap_{\alpha \in \Lambda} A_{\alpha}\right) \subseteq \frac{1}{n}\left(\bigcap_{\alpha \in \Lambda} A_{\alpha}\right)$.

Also we have the follwing corollary.

Corollary 4. For $x, y \in X$ where $(X, \Gamma)$ is a semi-linear uniform spaces, then $n\left(\frac{1}{n} \rho(x, y)\right) \subseteq \rho(x, y)$.

Corollary 5. For $n \in \mathbb{N}, V \in \Gamma$, if $\frac{1}{n} V \in \Gamma$, then $\frac{1}{n} V$ is the largest element in $\Gamma$ satisfies $n\left(\frac{1}{n} V\right) \subseteq V$.

Also by Definition 3.6, for $x \cdot y \in X$ where $(X, \Gamma)$ is a semi-linear uniform spaces, we have,

i) $\frac{1}{n} \rho(x, y)=\bigcap\left\{\frac{1}{n} V: V \in \Gamma_{(x, y)}\right\}$.

ii) $\frac{1}{n} \delta(x, y)= \begin{cases}\bigcup\left\{\frac{1}{n} V: V \in \Gamma_{(x, y)}^{c}\right\} & \text { if } x \neq y, \\ \phi & \text { if } x=y .\end{cases}$

Proposition 5. Let $x \cdot y$ be any distinct points in semi-linear uniform spaces $(X, \Gamma)$. Then, $n\left(\frac{1}{n} \delta(x, y)\right) \subseteq \delta(x, y) \subseteq \frac{1}{n}(n \delta(x, y))$.

Proof. Let $\left(s_{0}, s_{n}\right) \in n\left(\frac{1}{n} \delta(x, y)\right)$, then $\left(s_{0}, s_{n}\right) \in n \bigcup\left\{\frac{1}{n} V: V \in \Gamma_{(x, y)}^{c}\right\}$, then there exist $s_{1}, \cdots, s_{n-1}$ such that $\left(s_{j}, s_{j+1}\right) \in \bigcup\left\{\frac{1}{n} V: V \in \Gamma_{(x, y)}^{c}\right\}, j=0, \cdots, n-1$. So there exist $U_{1}, U_{2}, \cdots, U_{n} \in \Gamma_{(x, y)}^{c}$ such that $\left(s_{j-1}, s_{j}\right) \in \frac{1}{n} U_{j}$, but $\Gamma$ is a chain implies the existence of $j_{0} \in\{1, \cdots, n\}$ such that $U_{j} \subseteq U_{j_{0}}$ for all $j=1, \cdots, n$. So $\left(s_{0}, s_{n}\right) \in n\left(\frac{1}{n} U_{j_{0}}\right) \subseteq U_{j_{0}} \subseteq \delta(x, y)$. On the other hand by proposition 1.7, $n \delta(x, y)=\bigcup_{V \in \Gamma_{(x, y)}^{c}} n V$. So for all $V \in \Gamma_{(x, y)}^{c}, \quad n V \subseteq n \delta(x, y)$, which implies $V \subseteq \frac{1}{n}(n \delta(x, y))$.

Definition 6. For $n \in \mathbb{N}, A, A_{\alpha} \subseteq X \times X, \alpha \in \Lambda$. and $r \in \mathbb{Q}^{+}$, define i) $r A=n\left(\frac{1}{m} A\right), \quad r=\frac{n}{m}, n, m \in \mathbb{N}$ and the greatest common devisor of $n, m$ is 1 .

ii) $r \bigcup\left\{A_{\alpha}: \alpha \in \Lambda\right\}=\bigcup\left\{r A_{\alpha}: \alpha \in \Lambda\right\}$.

iii) $r \bigcap\left\{A_{\alpha}: \alpha \in \Lambda\right\}=\bigcap\left\{r A_{\alpha}: \alpha \in \Lambda\right\}$.

Definition 7. Let $x \cdot y$ be any points in semi-linear uniform spaces $(X, \Gamma)$. For $r \in \mathbb{Q}^{+}$, $r \delta(x, y)=n\left(\frac{1}{m} \delta(x, y)\right)$, where $r=\frac{n}{m}, n, m \in \mathbb{N}$ and the greatest common devisor of $n, m$ is 1 .

Using the a bove definition and Proposition 1.7, we have.

Proposition 6. Let $V \in \Gamma$. and $r_{1}, r_{2} \in \mathbb{Q}^{+}, r_{1}<r_{2}$, then $r_{1} V \subseteq r_{2} V$. 
Proposition 7. Let $x \cdot y$ be any points in semi-linear uniform spaces $(X, \Gamma)$. If $r_{1}, r_{2} \in \mathbb{Q}^{+}, r_{1} \leq r_{2}$, then $r_{1} \delta(x, y) \subseteq r_{2} \delta(x, y)$.

Definition 8. Let $r_{n}$ be an increasing sequence of positive rationals. If $r_{n} \rightarrow r \in \mathbb{Q}^{+}$, then

$\lim _{n \rightarrow \infty} r_{n} \delta(x, y)$ is defined by $\bigcup_{n=1}^{\infty} r_{n} \delta(x, y)$.

Proposition 8. Let $x, y \in(X, \Gamma)$ and $r_{n}$ an increasin sequence of positive rationals. If $r_{n} \rightarrow r \in \mathbb{Q}^{+}$, then $\lim _{n \rightarrow \infty} r_{n} \delta(x, y) \subseteq r \delta(x, y)$.

Proof. It is an immediate consequence of Proposition (7) and Definition (8).

\section{Contractions}

In [10] the definitions of converges and Cauchy are given. Now we shall discuss some topological properties of semi-linear uniform spaces. Since the semi-linear uniform space is a topological space then the continuity of a function is as in topology. The concept of uniform continuity is given by Wiels [4], so we have:

Definition 9 [4]. Let $f:\left(X, \Gamma_{X}\right) \rightarrow\left(Y, \Gamma_{Y}\right)$, then $f$ is uniformly continuous if $\forall U \in \Gamma_{Y}, \exists V \in \Gamma_{X}$, such that if $(x, y) \in V$, then $(f(x), f(y)) \in U$.

Clearly using our notation we have:

Proposition 9. Let $f:\left(X, \Gamma_{X}\right) \rightarrow\left(Y, \Gamma_{Y}\right)$. Then $f$ is uniformly continuous, if and only if $\forall U \in \Gamma_{Y}$, $\exists V \in \Gamma_{X}$ such that, for all $x, y \in X$, if $\rho_{X}(x, y) \subseteq V$, then $\rho_{Y}(f(x), f(y)) \subseteq U$.

The following proposition shows that we may replace $\rho$ by $\delta$ in Proposition 2.2.

Proposition 10 [12]. Let $f:\left(X, \Gamma_{X}\right) \rightarrow\left(Y, \Gamma_{Y}\right)$. Then $f$ is uniformly continuous, if and only if $\forall U \in \Gamma_{Y}$, $\exists V \in \Gamma_{X}$, such that for all $x, y \in X$, if $\delta_{X}(x, y) \subseteq V$, then $\delta_{Y}(f(x), f(y)) \subseteq U$.

In [10], Tallafha gave an example of a space which was the semi-linear uniform space, but not metrizable. Till now, to define a function $f$ that satisfies Lipschitz condition, or to be a contraction, it should be defined on a metric space to another metric space. The main idea of this paper is to define such concepts without metric spaces, and we just need a semi-linear uniform space, which is weaker as we mentioned before.

Definition 10 [1]. Let $f:(X, \Gamma) \rightarrow(X, \Gamma)$, then $f$ satisfied Lipschitz condition if there exist $m, n \in \mathbb{N}$ such that $m \delta(f(x), f(y)) \subseteq n \delta(x, y)$. Moreover if $m>n$, then we call $f$ a contraction.

Now we shall give a new definition of Lipschitz condition and contraction called $r$-Lipschitz condition and $r$-contraction.

Definition 11. Let $f:(X, \Gamma) \rightarrow(X, \Gamma)$, then $f$ satisfied $r$-Lipschitz condition if there exists $r \in \mathbb{Q}^{+}$such that $\delta(f(x), f(y)) \subseteq r \delta(x, y)$. Moreover if $r<1$ then we call $f$ a $r$-contraction.

Question. Let $(X, \Gamma)$ be semi-linear uniform spaces and $f:(X, \Gamma) \rightarrow(X, \Gamma)$, what is the relation between Lipschitz condition and $r$-Lipschitz condition, contraction, and r-contraction.

Remark 1 [12]. Let $(X, \Gamma)$ be semi-linear uniform spaces, then if $\Delta \in \Gamma$, then the topology indused by $(X, \Gamma)$ is the descrete topology which is metrizable. Therefore we can asumme that $\Delta \notin \Gamma$.

Question [1] [12]. Let $(X, \Gamma)$ be a complete semi-linear uniform space. And $f:(X, \Gamma) \rightarrow(X, \Gamma)$ be a contraction. Does $f$ has a unique fixed point?

Question. Let $(X, \Gamma)$ be a complete semi-linear uniform space. And $f:(X, \Gamma) \rightarrow(X, \Gamma)$ be $r$-contraction. Does $f$ has a unique fixed point?

\section{References}

[1] Tallafha, A. (2013) Open Problems in Semi-Linear Uniform Spaces. Journal of Applied Functional Analysis, 8, 223228.

[2] Weil, A. (1936) Les recouvrements des espaces topologiques: Espaces complete, espaces bicompact. Comptes Rendus de l'Académie des Sciences, 202, 1002-1005.

[3] Weil, A. (1937) Sur les espaces a structure uniforme et sur la topologie generale. Actuarial Science India, 551, Paris.

[4] Weil, A. (1938) Sur les espaces à structure uniforme et sur la topologic générale. Hermann, Paris.

[5] Cohen, L.W. (1937) Uniformity Properties in a Topological Space Satisfying the First Denumerability Postulate. Duke Mathematical Journal, 3, 610-615. 
[6] Cohen, L.W. (1939) On Imbedding a Space in a Complete Space. Duke Mathematical Journal, 5, 174-183.

[7] Graves, L.M. (1937) On the Completing of a Housdroff Space. Annals of Mathematics, 38, 61-64.

[8] Bourbaki, N. (1940) Topologie Générale (General Topology). Paris.

[9] James, I.M. (1987) Topological and Uniform Spaces. Undergraduate Texts in Mathematics. Springer-Verlag, Berlin.

[10] Tallafha, A. and Khalil, R. (2009) Best Approximation in Uniformity Type Spaces. European Journal of Pure and Applied Mathematics, 2, 231-238.

[11] Tallafha, A. (2011) Some Properties of Semi-Linear Uniform Spaces. Boletin da Sociedade Paranaense de Matematica, 29, 9-14.

[12] Tallafha, A. (2015) Fixed Point in a Non-Metrizable Spaces. To Appear.

[13] Engelking, R. (1968) Outline of General Topology. North-Holand, Amsterdam. 